\title{
ESPECTROMETRIA DE ABSORÇÃO ATÔMICA: O CAMINHO PARA DETERMINAÇÕES MULTI- ELEMENTARES
}

\author{
Fábio Alan Carqueija Amorim \\ Instituto de Ciências Ambientais e Desenvolvimento Sustentável, Campus Univ. Prof. Edgard Santos, Universidade Federal da \\ Bahia, 47805-100 Barreiras - BA; Departamento de Química Analítica, Instituto de Química, Campus Univ. de Ondina, Universidade \\ Federal da Bahia, 40170-290 Salvador - BA, Brasil \\ Ivon Pinheiro Lobo \\ Universidade Estadual de Santa Cruz, Rodovia Ilhéus/Itabuna, km 16, 45662-000 Ilhéus - BA; Departamento de Química Analítica, \\ Instituto de Química, Campus Univ. de Ondina, Universidade Federal da Bahia, 40170-290 Salvador - BA, Brasil \\ Vera Lucia C. S. Santos e Sérgio Luis Costa Ferreira* \\ Departamento de Química Analítica, Instituto de Química, Campus Univ. de Ondina, Universidade Federal da Bahia, \\ 40170-290 Salvador - BA, Brasil
}

Recebido em 23/3/07; aceito em 19/12/07; publicado na web em 11/9/08

\begin{abstract}
ATOMIC ABSORPTION SPECTROMETRY: THE WAY FOR MULTIELEMENT DETERMINATIONS. This paper present an overview of way covered for the spectrometry of atomic absorption (AAS), tracing a line of the historical events in its development and its establishment as a multielement technique. Additionally, the efforts carried by through several researchers in the search for the instrumental evolution, the advances, advantages, limitations, and trends of this approach are related. Several works focusing its analytical applications are cited employing simultaneous multielement determination by flame (FAAS) and/or graphite furnace (GF AAS), and fast sequential multielement determination using FAAS are reported in the present review.
\end{abstract}

Keywords: multielement AAS; simultaneous determination; fast sequential FAAS.

\section{INTRODUÇÃO}

Considerada como uma técnica analítica bem sucedida, a espectrometria de absorção atômica (AAS - Atomic Absorption Spectrometry) é uma das mais utilizadas na determinação de elementos em baixas concentrações, que estão presentes numa variedade de amostras, sejam estas líquidas, sólidas, em suspensão, e até mesmo gasosas, podendo estar associada a sistemas de análise em fluxo e permitir estudos de especiação. ${ }^{1,2}$

Todas as aplicações analíticas modernas da AAS tiveram origem a partir de trabalhos publicados no final da década de $50,,^{3,4}$ que propuseram pela primeira vez o uso da AAS para análises químicas. ${ }^{5}$ Marcando, em 2005, o aniversário de 50 anos de existência desta publicação, deve-se reconhecer que o uso da AAS muito contribuiu para o progresso científico neste meio século. ${ }^{6-10}$ Entretanto, deve-se também reconhecer que esta técnica apresenta limitações, particularmente quando comparada com a espectrometria de emissão óptica com plasma indutivamente acoplado (ICP OES - Inductively Coupled Plasma Optical Emission Spectrometry) e a espectrometria de massa com plasma indutivamente acoplado (ICP-MS - Inductively Coupled Plasma Mass Spectrometry), que são conceitualmente estabelecidas como técnicas multi-elementares, característica que não é atribuída à AAS. ${ }^{11,12}$

Neste sentido, apresenta-se a seguir uma revisão envolvendo o uso da AAS para determinações multi-elementares, registrando os esforços realizados por diversos pesquisadores na busca pelo desenvolvimento instrumental, apontando as tendências evolutivas desta técnica, focalizando suas aplicações analíticas.

\section{ABSORÇÃO ATÔMICA: O INÍCIO}

A espectrometria atômica teve papel fundamental no desenvolvimento da química. Esta página da história teve início com os experimentos de Joannes M. Marci, em 1648, através de suas observações a respeito da origem do arco-íris com bases no espalhamento da luz solar nas gotículas d'água. ${ }^{1}$ Mais tarde, em 1672, Isaac Newton descrevia a decomposição e recomposição da luz do sol por um prisma. Daí por diante, vários experimentos foram realizados com o espectro oriundo da decomposição da luz solar, a exemplo da descoberta da região do infravermelho pelo inglês William Herschel, em 1800, e a região do ultravioleta em 1801, pelo alemão Johann W. Ritter e o inglês William H. Wollaston. ${ }^{13}$ Wollaston também descobriu as linhas escuras no espectro obtido a partir da luz do sol; linhas essas que foram explicadas em 1820 por Brewster como sendo ocasionadas pelo processo de absorção da radiação na atmosfera do sol. ${ }^{1}$ Joseph Fraunhofer observou os espectros de linhas emitidos por alguns elementos químicos. Em 1859, o químico Robert W. Bunsen em parceria com o físico Gustav R. Kirchhoff criaram um espectroscópio que lhes permitia observar as linhas de emissão de elementos químicos que eram excitados em uma chama não luminosa (queimador de Bunsen). Com o uso desse equipamento, descobriram dois novos elementos químicos, o césio e o rubídio. ${ }^{13}$

Desde a descrição do processo de emissão e absorção de energia radiante por átomos na chama por Kirchhoff e Bunsen em $1860,{ }^{1}$ a emissão atômica prendeu a atenção dos pesquisadores, sendo escolhida como técnica analítica na determinação de metais, principalmente na indústria metalúrgica. Nestes quase 100 anos, o fenômeno da absorção de energia radiante por átomos livres no estado gasoso presentes em uma chama foi então esquecido, até mesmo pela falta de componentes necessários para o avanço das pesquisas, até que em 1954 o cientista australiano Alan Walsh propôs uma técnica analítica 
para determinação de metais, a espectrometria de absorção atômica em chama (FAAS - Flame Atomic Absorption Spectrometry), baseada justamente neste fenômeno, e que só foi completamente aceita a partir de 1960. ${ }^{14}$ Até então, a comunidade científica não demonstrou grande interesse pelo trabalho de Walsh, com exceção do cientista russo Boris L'vov, que, 2 anos após Walsh ter apresentado pela primeira vez a espectrometria de absorção atômica como técnica elementar, propôs um espectrômetro de absorção atômica que utilizava um forno de grafite como atomizador eletrotérmico (GFAAS - Graphite Furnace Atomic Absorption Spectrometry), ${ }^{15}$ obtendo assim, uma redução bastante significativa dos limites de detecção. ${ }^{16}$

Inicialmente, Walsh levantou a possibilidade de utilizar uma fonte contínua de radiação, porém, seriam necessários monocromadores de alta resolução para isolar as linhas espectrais e garantir a relação entre a absorção e a concentração, ${ }^{14,17}$ o que na época era impraticável. Walsh propôs então o uso de lâmpadas de emissão de linhas espectrais atômicas, cujas larguras são mais estreitas que as linhas de absorção. Foram empregadas lâmpadas de catodo oco (HCL - hollow cathode lamps) como fontes de linha. Estas lâmpadas foram propostas inicialmente por Paschen, em 1916, porém, naquela época eram instáveis e apresentavam dificuldades de operação, pois necessitavam de uma atmosfera de argônio de $1 \mathrm{KPa}$ que deveria ser continuamente bombeada. A solução encontrada por Walsh e colaboradores foi confeccionar as HCLs em tubos selados de vidro com janela de quartzo. As HCLs foram, então, peças fundamentais para o desenvolvimento do espectrômetro de absorção atômica, dispensando o emprego de monocromadores de alta resolução. ${ }^{17}$

Ao longo dos anos foram ocorrendo novos avanços que contribuíram para o desenvolvimento da AAS, a exemplo da câmara de pré-mistura acoplada aos queimadores dos espectrômetros de chama, da lâmpada de catodo oco de alta intensidade, ${ }^{16}$ da introdução do conceito STPF (stabilized temperature platform furnace), por Slavin et al. ${ }^{18}$ Ocorreram também pesquisas e aplicação de novas fontes de radiação como as lâmpadas de descarga sem eletrodo (EDL - electrodeless discharge lamps), HCLs multi-elementares e o uso de laser de diodos. ${ }^{1}$ As lâmpadas multi-elementares foram propostas e muito usadas em meados da década de 60. Estas só são aplicáveis a grupos de elementos com propriedades similares, desde que os elementos combinados na lâmpada não apresentem interferências espectrais entre si. Por causa das suas intensidades de emissão reduzidas, comparadas com as HCLs mono-elementares, estas fontes de radiação não obtiveram ampla aplicação. ${ }^{19}$

A espectrometria de absorção atômica atraiu bastante interesse, mostrado pelo crescente número de publicações a partir de 1960, devido a sua simplicidade e baixo custo de operação. Este interesse é mais evidenciado analisando-se o número de laboratórios que adquiriram este equipamento, principalmente os laboratórios industriais. ${ }^{20}$

Com o desenvolvimento, a passos largos, da espectrometria de emissão óptica com plasma indutivamente acoplado (ICP OES) e da espectrometria de massa com plasma indutivamente acoplado (ICPMS), as vantagens e desvantagens de cada técnica passaram a ser comparadas. A AAS apresenta algumas vantagens quando comparada com a técnica de emissão óptica, tais como, menor custo do equipamento, menor custo operacional, facilidade de operação, além do reduzido número de linhas espectrais, que acarreta uma possibilidade bem menor de interferência por sobreposição de linhas. Quanto às desvantagens, a espectrometria de absorção atômica apresenta faixa linear de trabalho relativamente pequena, menor freqüência analítica quando se utiliza atomização eletrotérmica, elementos não metálicos como fósforo e enxofre não são determinados facilmente e, principalmente, é considerada uma técnica analítica mono-elementar, fato que é reconhecido como a maior desvantagem. ${ }^{11}$

Com base no desenvolvimento da ICP OES, começaram a apare- cer previsões a respeito do abandono da AAS como técnica analítica. Em 1989, Hieftje ${ }^{21}$ publicou uma revisão onde demonstrou a redução no número de publicações acadêmicas que faziam uso da espectrometria de absorção atômica, a partir daí chamou a atenção a respeito da possibilidade desta técnica analítica cair em desuso e ser retirada do mercado até o ano de 2000, caso esta tendência continuasse e se não houvesse novos avanços tecnológicos.

Atualmente, verifica-se que a AAS é ainda bastante empregada e que ganha bastante força com o emprego de técnicas de injeção em fluxo e possibilidade de separação e pré-concentração on-line. ${ }^{16}$ Segundo Sneddon e colaboradores, ${ }^{12}$ o uso e o interesse pela AAS não são representados pelo número de publicações, que são indicativos do interesse acadêmico, mas sim, pelo número de laboratórios que fazem uso desta técnica.

\section{DETERMINAÇÕES MULTI-ELEMENTARES USANDO AAS}

A busca por determinações multi-elementares utilizando AAS teve início cedo, se comparada com a apresentação desta técnica por Walsh, a começar pelos trabalhos de Butler e Strasheim, em 1965, e de Sullivan e Walsh em $1967 .{ }^{1}$ Sneddon et al. ${ }^{12}$ apresentaram, em artigo de revisão, um enfoque da perspectiva histórica da AAS multi-elementar. Durante todo o desenvolvimento histórico, as determinações multi-elementares por AAS foram realizadas unicamente de forma simultânea. Apenas 50 anos após a implementação da AAS como técnica analítica, determinações multi-elementares realizadas de forma seqüencial vieram a ser realizadas.

A seguir, são relatados diversos trabalhos realizados por meio de determinações multi-elemetares simultâneas e seqüenciais, separadamente.

\section{Determinação multi-elementar simultânea}

Várias tentativas foram realizadas para construir um espectrômetro de absorção atômica multi-elementar, utilizando um arranjo de lâmpadas de catodo oco ou de descarga sem eletrodo, mono ou policromadores, atomizadores de chama e detectores convencionais. Estes instrumentos foram caracterizados pela baixa sensibilidade, pobres razões sinal/ruído e inflexibilidade quanto os elementos a serem determinados. Nesta linha de trabalho, Aldous et al., ${ }^{22} \mathrm{em}$ 1975, realizaram determinações simultâneas de sete metais em água potável utilizando um espectrômetro de absorção atômica multicanal, equipado com duas lâmpadas de catodo oco multi-elementares. O desempenho deste equipamento, no entanto, foi inferior ao de um equipamento convencional. Hoje, sabe-se que as combinações de lâmpadas de catodo oco mono e multi-elementares sofrem de perda da intensidade devido ao sistema de divisão e combinação do feixe de radiação. ${ }^{23}$

A busca por uma solução que implicasse em um conceito mais flexível fez com que as atenções dos pesquisadores se voltassem, mais uma vez, para as fontes de radiação contínua. Embora a fonte contínua possa operar nas regiões ultravioleta e visível do espectro eletromagnético, a largura de banda isolada com monocromadores de média resolução, comumente empregados na AAS convencional, ainda é muito maior que as obtidas com fontes de linha. Esta largura de banda resulta na diminuição de sensibilidade e especificidade, curvas de calibração não lineares e grande susceptibilidade à interferência da radiação de fundo. Outros problemas observados quando se utilizam fontes contínuas são linhas de base com muito ruído e limites de detecção relativamente mais elevados, que são relacionados com a instabilidade das fontes contínuas mais intensas, a exemplo das lâmpadas de arco do xenônio; ${ }^{19,23}$ além disso, a intensidade das fontes contínuas diminui consideravelmente 
em comprimentos de onda abaixo de $280 \mathrm{~nm}$, portanto, não se pode simplesmente substituir as lâmpadas de catodo oco por fonte de radiação contínua sem adaptações no restante do instrumento.

Dispostos a obterem linhas mais estreitas a partir de uma fonte contínua, no caso uma lâmpada de arco de xenônio de alta intensidade, O'Haver e colaboradores, em 1976, empregaram um policromador de Echelle de alta resolução para separação de linhas espectrais com larguras de bandas mais estreitas. ${ }^{1}$ Harnly e colaboradores,${ }^{24} \mathrm{em} 1979$, empenharam-se no desenvolvimento, caracterização e promoção da fonte contínua. ${ }^{25,26}$ Eles desenvolveram um espectrômetro de absorção atômica com fonte contínua para determinações multi-elementares simultâneas (SIMAAC - Simultaneous Multielement Atomic Absorpition Continuum), compatível com atomizadores de chama ou eletrotérmicos, utilizando policromador Echelle modificado, uma lâmpada de arco de xenônio como fonte contínua de radiação e um sistema computadorizado para aquisição de dados. ${ }^{27}$ Para minimizar a oscilação da intensidade da lâmpada de arco de xenônio foi empregada modulação mecânica do comprimento de onda, utilizando um refrator de quartzo acoplado a motor de torque. Este sistema permitia a leitura de intensidades fora da linha analítica para corrigir deslocamentos largos de faixa na intensidade espectral, causados pela oscilação da lâmpada ou por absorção não específica de fundo. No entanto, esse conjunto não conseguiu operar em frequiências acima de $60 \mathrm{~Hz}$ sem sacrificar o sinal em relação ao ruído $(\mathrm{S} / \mathrm{N}) .{ }^{23}$ Utilizando este equipamento determinaram simultaneamente 9 elementos, empregando atomizadores de chama, para isso, estudaram 16 diferentes combinações de altura do queimador e da estequiometria da chama. Com o emprego de detectores de estado sólido, a partir de 1990, eliminou-se a necessidade da modulação mecânica do comprimento de onda e possibilitou-se a leitura simultânea em diferentes comprimentos de onda.

Em 1985, Carroll et al. ${ }^{28}$ propuseram o uso de uma sonda de grafite em conjunto com o sistema de atomização eletrotérmica de um SIMAAC, o que conferiu uma atomização isotérmica na determinação de 15 elementos (Al, Ca, Cd, Co, Cr, Cu, Fe, K, Mg, Mn, Mo, Ni, Pb, $\mathrm{V}$ e Zn). Uma temperatura de atomização de $2700^{\circ} \mathrm{C}$ foi estabelecida para uma eficiente atomização e melhores sensibilidades para todos os elementos estudados. Comparando os resultados obtidos por este sistema com os obtidos por atomização em tubo e em plataforma, melhores resultados foram obtidos para os elementos mais voláteis, no entanto, para os elementos mais refratários (ex. V e Mo) os resultados foram menos satisfatórios.

Em 1992, Farah e Sneddon ${ }^{29}$ realizaram estudo de otimização das condições de operação de um espectrômetro de absorção atômica Smith-Hieftje 8000 (Thermo Jarrell Ash, Franklin, MA) com atomizador de chama, para quatro elementos ( $\mathrm{Mn}, \mathrm{Cu}, \mathrm{Zn}$ e Fe), utilizando otimização multivariada simplex. A capacidade de detecção multielementar deste equipamento baseia-se no uso de um monocromador contendo um sistema dirigido por um galvanômetro, com espelhos montados sobre um sistema mecânico que tem posicionamento ajustado pela aplicação de corrente elétrica. Este mecanismo permite que o instrumento faça a varredura de 190 a $800 \mathrm{~nm}$ em 20 ms. Para seleção das lâmpadas de catodo oco, foi utilizado um espelho também montado em um galvanômetro, possibilitando a análise multi-elementar e em uma única aspiração de amostra. Os parâmetros estudados foram: a estequiometria da chama (razão ar/acetileno), a altura do queimador, a largura da janela e a corrente das lâmpadas para cada elemento. Foram comparados os desempenhos do equipamento na determinação simultânea e mono-elementar, sendo acusada redução da sensibilidade na determinação dos elementos quando o espectrômetro foi operado no modo de determinação simultânea.

Fazendo uso deste mesmo tipo de equipamento Sneddon e colaboradores realizaram diversos trabalhos, dos quais se podem destacar três. No primeiro, ${ }^{30} \mathrm{em} 1993$, formam determinados 9 metais em amostras de soluções empregadas em revestimento eletrolítico, sendo os metais divididos em dois grupos, de acordo com suas características termoquímicas. Os resultados foram comparados sem utilizar correção de fundo, com correção de fundo de Smith-Hieftje e com correção com lâmpada de deutério. A correção de fundo de Smith-Hieftje baseia-se na subtração das absorvâncias medidas alternadamente com o funcionamento das lâmpadas na corrente usual e pulsada para corrente alta. Quando uma corrente elevada passa momentaneamente através do cátodo produz uma nuvem densa de átomos neutros que se aglomeram na saída da lâmpada. Isto permite que o sinal de fundo seja medido e subtraído do sinal da amostra. De acordo com o trabalho, foi observado que os melhores resultados foram obtidos utilizando correção de fundo de Smith-Hieftje. Segundo os autores, a correção de Smith-Hieftje foi mais eficiente quando a linha espectral estudada sofria influência do efeito de rollover. Os resultados sem correção de fundo foram inferiores aos obtidos por ambos os sistemas de correção empregados. O segundo, ${ }^{31}$ em 1995, foi aplicado para determinação de cádmio e chumbo em amostras de referência de sangue utilizando di-hidrogenofosfato de amônio e nitrato de magnésio como modificadores químicos. Em outro trabalho, um novo incremento foi aplicado para a determinação de $\mathrm{Cd}$ e $\mathrm{Pb}$ em cigarro e fumaça de cigarro. A fumaça foi coletada via uma simples etapa de impactação do aerossol sobre o tubo de grafite (I-GF AAS Impactation-graphite furnace atômic absorption spectrometry) a um fluxo e tempo fixados, seguido da medida analítica. Uma descrição mais detalhada sobre o sistema de impactação é discutida em outros trabalhos. ${ }^{2,32,33}$

A influência da geometria do forno de grafite na determinação simultânea de $\mathrm{Cd}, \mathrm{Co}, \mathrm{Cu}$ e $\mathrm{Pb}$ foi avaliada por Hoenig e Cillissen ${ }^{34}$ em 1997, os quais utilizaram um equipamento SIMAA 6000 da Perkin Elmer. Neste estudo, verificou-se que tubos de grafite com aquecimento longitudinal não são os mais adequados para a determinação de elementos voláteis, visto que estes tendem a migrar para as extremidades mais frias acarretando dispersão da nuvem atômica e, possivelmente, recombinações. Em 1998, Hammond et al. ${ }^{35}$ determinaram $\mathrm{Cd}, \mathrm{Pb}$ e $\mathrm{Ni}$ em óleo cru utilizando FAAS.

Gomes Neto e colaboradores, ${ }^{36}$ em 2001, determinaram $\mathrm{Cd}$ e $\mathrm{Pb}$ em vinhos branco e tinto por espectrometria de absorção atômica com atomização eletrotérmica (ET AAS - Electrothermal Atomic Absorpition Spectrometry) usando o equipamento SIMAA 6000 da Perkin Elmer e como atomizador um tubo de grafite transversalmente aquecido e efeito Zeeman longitudinal para correção da linha de base. Com o emprego da mistura da Pd e Mg como modificador químico foi evitada a formação de resíduos carbonáceos. Myöhänen et al. ${ }^{37}$ determinaram $\mathrm{As}, \mathrm{Cd}, \mathrm{Cr}$ e $\mathrm{Pb}$ em amostras de solos e de sedimentos, após digestão com água-régia, utilizando ET AAS (SIMAA 6000 da Perkin Elmer), com programa de aquecimento sem etapa de pirólise e sem utilizar modificadores químicos. Segundo os autores, foram obtidos resultados satisfatórios de exatidão e precisão. No entanto, foi observado um considerável efeito de memória para o cromo, que foi solucionado com emprego de altas temperaturas, culminando na redução da vida útil do tubo de grafite. Oliveira et al. realizaram determinação de Mn e Se em soro por ET AAS (SIMAA 6000), ${ }^{38}$ utilizando uma mistura de $\mathrm{Pd}$ e $\mathrm{Mg}\left(\mathrm{NO}_{3}\right)_{2}$ como modificador químico. Fizeram uso de uma mistura oxidante de $\mathrm{H}_{2} \mathrm{O}_{2} 15 \%(\mathrm{~m} / \mathrm{m})$ e $\mathrm{HNO}_{3} 1 \%$ (v/v), associada a uma etapa preliminar de pirólise, a $400{ }^{\circ} \mathrm{C}$, tornando mais eficiente a decomposição da parte orgânica; realizaram também a determinação direta de cromo e manganês em urina. ${ }^{39}$

Em 2003, Carrión e colaboradores ${ }^{40}$ determinaram $\mathrm{Pb}, \mathrm{Ni}, \mathrm{Sn}$ e $\mathrm{Cu}$ em liga de alumínio usando ET AAS. A análise foi realizada em uma suspensão coloidal, obtida através de descarga elétrica em meio aquoso. Conforme os resultados obtidos, o alumínio atuou como 
modificador químico estabilizando o $\mathrm{Pb}$ e o $\mathrm{Sn}$, permitindo alcançar temperaturas de pirólise de 1100 e $1300{ }^{\circ} \mathrm{C}$, respectivamente. Chiu et al.$^{41}$ determinaram traços de $\mathrm{Mn}, \mathrm{Fe}$, e Co em cobre por ET AAS (SIMAA 6000), após digestão assistida por microondas. Utilizaram $\mathrm{Pd}\left(\mathrm{NO}_{3}\right)_{2}$ como modificador químico e uma mistura de $95 \%$ de argônio e $5 \%$ de hidrogênio como gás de purga.

Nomura et al., ${ }^{42}$ em 2004, estudaram as interferências de $\mathrm{Na}^{+}$, $\mathrm{K}^{+}, \mathrm{Ca}^{2+}, \mathrm{Mg}^{2+}, \mathrm{Cl}^{-}, \mathrm{NO}_{3}^{-}, \mathrm{SO}_{4}^{2-}, \mathrm{PO}_{4}^{3-}$ na atomização simultânea de $\mathrm{As}, \mathrm{Cd}, \mathrm{Pb}$ e Se, nas determinações em espectrometria de absorção atômica com atomização eletrotérmica em forno de grafite, utilizando W e Rh como modificadores químicos permanentes.

Encontram-se disponíveis na literatura inúmeros trabalhos que descrevem o desenvolvimento e as aplicações de métodos para determinação de diferentes elementos nos mais diversos tipos de amostras por meio de AAS multi-elementar simultânea. ${ }^{43-61}$ A Tabela 1 apresenta vários trabalhos publicados nos últimos 20 anos relacionados à aplicação da AAS multi-elementar simultânea.

As possibilidades oferecidas pela ET AAS na determinação multielementar simultânea, tais como, excelentes limites de detecção para a maioria dos elementos, possibilidade de análise direta, necessidade de pequenos volumes de amostra, alta frequiência analítica, baixo custo operacional e a possibilidade do uso de padronização interna, implicaram em um maior número trabalhos com esta técnica analítica que com atomizadores de chama. ${ }^{65}$ Uma das grandes dificuldades, porém, tem sido o estabelecimento de um programa de aquecimento e a escolha de um modificador químico adequado e que atenda a determinação simultânea em ET AAS, garantindo a precisão, exatidão e repetibilidade das medidas. Sneddon et al. ${ }^{12}$ anteciparam que sistemas de determinação multi-elementar utilizando absorção atômica só seriam capazes de determinar, no máximo, seis elementos, isto devido ao grau de dificuldade em se montar um equipamento contendo todos os dispositivos necessários para processar os resultados das determianções.

Em seu artigo de revisão, Welz et al. ${ }^{14}$ apresentaram uma visão crítica do novo conceito instrumental para AAS. Trata-se da espectrometria de absorção atômica de alta resolução com fonte contínua (HR-CS AAS - High-Resolution Continuum-source Atomic Absorption Spectrometry). ${ }^{63} \mathrm{~A}$ fonte de radiação utilizada foi uma lâmpada de arco curto de xenônio de alta intensidade e, para separação das linhas, foi utilizado um monocromador de alta resolução double-echelle e um dispositivo com arranjo de carga acoplado como detector, permitindo uma resolução de aproximadamente $2 \mathrm{pm}$ por pixel. Além do alto poder de resolução, que possibilita distinguir linhas espectrais, o que até então não era possível utilizando a AAS convencional, a HR-CS AAS registra 60 espectros $\mathrm{s}^{-1}$ e permite a "visualização do ambiente espectral" da linha do analito, tornando possível, desta forma, a correção da variação da intensidade da fonte, da absorção de fundo, das interferências devido à sobreposição de linhas e dos espectros estruturados. Dentre outras vantagens que também foram anunciadas estão uma melhora na razão sinal/ruído devido à elevada intensidade da fonte de radiação, resultando em melhor precisão e limites de detecção e novos elementos podem ser determinados, para os quais fontes de radiação ainda não são disponíveis.

\section{Determinação multi-elementar seqüencial}

Embora a determinação multi-elementar simultânea seja de grande interesse, principalmente com a evolução e aplicação dos sistemas de alta resolução com fonte contínua (HR-CS AAS), a limitação no que diz respeito ao comportamento termoquímico bastante distinto entre os diversos elementos, particularmente com atomizadores eletrotérmicos, ainda é crítica. Nesse sentido, determinações multi-elementares seqüenciais podem ser realizadas utilizando atomizadores de chama, ${ }^{64}$ técnica que se apresenta como uma atraente alternativa para a AAS.
Além do comprimento de onda desejado, que é selecionado de forma rápida pelo sistema do monocromador, outras condições de operação, como a estequiometria da chama e a altura do queimador, podem ser selecionadas de forma independente para cada elemento. Projahn et al. ${ }^{65} \mathrm{em} 2004$, estudaram uso do método de elemento de referência para determinação multi-elementar seqüencial utilizando um espectrômetro de absorção atômica com atomizador de chama SpectrAA 220 FS da Varian (FS FAAS - Fast Sequential Atomic Absorption Spectrometry). Este equipamento utiliza um monocromador com velocidade de varredura de $33 \mathrm{~nm} \mathrm{~s}^{-1}$, lâmpadas de catodo oco como fonte de radiação, que são operadas simultaneamente e selecionadas utilizando-se um espelho motorizado. A estequiometria da chama é ajustada através do controle automático programável do fluxo do gás. Segundo os autores, a frequiência analítica do FS FAAS excede a de um ICP OES seqüencial. Wang et $a l .{ }^{66,67}$ utilizaram também um espectrômetro SpectrAA 220 FS da Varian para determinação multi-elementar seqüencial de metais, após etapa preliminar de pré-concentração em coluna. Niedzielski ${ }^{68}$ apresentou um novo conceito de sistema analítico hifenado para especiação de arsênio e selênio inorgânicos utilizando cromatografia líquida de alta performance acoplada à detecção por AAS com geração de hidretos funcionando no modo seqüencial (HPLC-HG FS AAS). Este método foi aplicado na análise de amostras de água. A atomização foi realizada em um tubo de quartzo aquecido eletricamente à temperatura de $900^{\circ} \mathrm{C}$.

Nosso grupo de pesquisa vem se empenhando na realização de trabalhos envolvendo aplicações da AAS multi-elementar seqüencial. ${ }^{69,70}$ Amorim e Ferreira ${ }^{71}$ realizaram a determinação seqüiencial de cádmio e chumbo em sal de consumo humano, após etapa de separação e pré-concentração destes metais por meio de extração dos complexos metal-ditizona com metil-isobutil-cetona (MIBK), aspirando a fase orgânica diretamente para a chama. Utilizou-se um espectrômetro SpectrAA 220 FS, sem controle automático da altura do atomizador de chama; portanto, a altura de observação na chama foi estabelecida como condição de compromisso e fixada em 13,5 mm. Em outro trabalho, ${ }^{72}$ determinou-se zinco e manganês em amostras de chocolate em pó, explorando as vantagens da amostragem em suspensão para análise direta sem necessidade da etapa de digestão da amostra, minimizando assim o tempo de análise e o trabalho operacional, bem como a diminuição de riscos de contaminação e de perdas de analitos. Em outra aplicação, Amorim e colaboradores ${ }^{73}$ realizaram a determinação seqüencial de metais em amostras de óleo lubrificante usado. Mais recentemente, a FS FAAS foi utilizada para realizar determinações multi-elementares em procedimentos analíticos envolvendo calibração com elemento de referência, inicialmente proposto por Da Silva et al. ${ }^{74}$ que aplicaram essa metodologia para determinação seqüencial de ferro em etanol combustível hidratado utilizando níquel como elemento de referência. Outra aplicação do método de calibração com elemento de referência foi na determinação seqüencial e direta de ferro e manganês em amostras de vinho por FS FAAS, ${ }^{75}$ onde se testou a eficiência do cobalto, prata, níquel e índio na correção de efeito da matriz buscando uma minimização no tempo e no custo da análise pela eliminação da etapa de digestão da amostra, sendo o cobalto e o índio os mais eficientes.

Embora existam poucos trabalhos publicados, a determinação multi-elementar seqüencial utilizando FAAS já figura como um avanço de grande importância. Uma das vantagens desta técnica é o ganho bastante expressivo de frequiência analítica aliada ao fato de não precisar de condições de compromisso, assim como ocorre com a determinação simultânea em ET AAS. ${ }^{69}$

\section{CONCLUSÃO}

No início, as lâmpadas de catodo oco surgiram como uma solução elegante para a falta de tecnologia que permitisse o uso de fontes 
Tabela 1. Aplicações de métodos analíticos utilizando AAS multi-elementar simultânea, publicados nos últimos 20 anos

\begin{tabular}{lc}
\hline Analitos & \multicolumn{1}{c}{ Amostra } \\
\hline $\mathrm{Ca}$ e $\mathrm{Mg}$ & Água \\
$\mathrm{Al}, \mathrm{Cu}, \mathrm{Fe}, \mathrm{Mn}, \mathrm{Cr}, \mathrm{Se}$, & Material particulado aéreo \\
$\mathrm{As}, \mathrm{Pb}, \mathrm{Ni}, \mathrm{Zn}$ e $\mathrm{Cd}$ & Carbeto e nitrato de silício \\
$\mathrm{Al}, \mathrm{Ca}$ e $\mathrm{Fe}$ & \\
$\mathrm{Pb}, \mathrm{Ni}, \mathrm{Sn}$ e $\mathrm{Cu}$ & Ligas à base de alumínio \\
$\mathrm{Ni}$ & Águas \\
$\mathrm{As} \mathrm{e} \mathrm{Se}$ & Urina \\
$\mathrm{Se}$ & Leite
\end{tabular}

As, $\mathrm{Cd}, \mathrm{Pb}$ e $\mathrm{Se} \quad$ Soluções de eletrólitos

$\mathrm{Pb}$

$\mathrm{Cd} \mathrm{e} \mathrm{Pb}$

$\mathrm{Al}, \mathrm{As}, \mathrm{Cu}, \mathrm{Fe}, \mathrm{Mn}$ e Ni

$\mathrm{As}, \mathrm{Cd}, \mathrm{Cr}$ e $\mathrm{Pb}$

Cr, Mo e V

$\mathrm{Pb}$

$\mathrm{Cu}, \mathrm{Cd}$ e $\mathrm{Pb}$

Mo e V

$\mathrm{Cr}, \mathrm{Cd}$, Ni e Pb

Co e Mn

Se e As

$\mathrm{Ru}, \mathrm{Rh}, \mathrm{Pt}, \mathrm{Ir}, \mathrm{Pd}, \mathrm{Ag}$ e $\mathrm{Au}$

Y, Nd, Sm, Sc, Eu, Dy,

Ho, Er, Tm e Yb

Etanol combustível

Solos e sedimentos

Telurito de bismuto

$\left(\mathrm{Bi}_{2} \mathrm{TeO}_{5}\right)$

Água de consumo

Água de mar

Urina

Água

Rochas silicatadas e minérios

Materiais de referência geológicos
Comentários

Ref.

Análise direta por FAAS com detector de carga acoplada

Urina, sangue e placenta

Fluidos do corpo humano (CCD, - coupled changer detector) e lâmpadas de cátodo oco como fonte de linha

Amostragem em suspensão com as partículas e detecção

$$
\text { por ET AAS }
$$

Amostragem em suspensão do material pulverizado e detecção por GF AAS

Suspensão coloidal metálica obtida por uma descarga elétrica operada no meio líquido

Determinação por GF AAS com aquecimento transversal (TH - transversely heated), uso de Co como padrão interno e $\mathrm{Mg}\left(\mathrm{NO}_{3}\right)_{2}$ como modificador químico

Avaliação do Co e do Sn como padrão interno e utilização de Pd e Mg como modificadores químicos

As e Ge como padrão interno em tubo de grafite pré-tratado com $\mathrm{W}$

$\mathrm{W}+\mathrm{Rh}$ depositado sobre o tubo de grafite e usado como modificador químico permanente

Bi usado como padrão interno e $\mathrm{Pd}\left(\mathrm{NO}_{3}\right)_{2}+\mathrm{Mg}\left(\mathrm{NO}_{3}\right)_{2}$ como modificadores químicos em tubo de grafite aquecido transversalmente

Análise direta por THGF AAS usando $\mathrm{NH}_{4} \mathrm{H}_{2} \mathrm{PO}_{4}$ como modificador químico

Uso de W-Rh como modificador permanente

combinado com os modificadores convencionais $\mathrm{Pd}\left(\mathrm{NO}_{3}\right)_{2}$

$$
+\mathrm{Mg}\left(\mathrm{NO}_{3}\right)_{2} \text { e detecção por THGF AAS }
$$

Digestão com água-régia e uso de HTGF AAS com correção longitudinal de background por efeito Zeeman

Matriz dissolvida em ácido clorídrico, modificada por triamônio citrato e detecção por HTGF AAS

Uso de Bi e Tl como padrões internos

Determinação por AAS com filamento de tungstênio e CCD

Avaliação dos modificadores $\mathrm{Mg}\left(\mathrm{NO}_{3}\right)_{2}, \mathrm{Pd}\left(\mathrm{NO}_{3}\right)_{2} \mathrm{e}$ mistura de ambos

Amostra diluída em $\mathrm{HNO}_{3}$ 0,1\%/Triton X-100 0,1\% e $\mathrm{NH}_{3}-\mathrm{Na}$ EDTA/ $\mathrm{NH}_{4} \mathrm{H}_{2} \mathrm{PO}_{4}$ como modificadores químicos

Amostra diluída em $\mathrm{HNO}_{3} 2 \%$ e Triton X-100 0,05\%, 1:1

Geração de hidretos e atomização em tubo de grafite recoberto por $\mathrm{Zr}$

Ataque com ácido fluorídrico e água-régia seguido por pré-concentração por cromatografia de troca-iônica

Dissolução ácida e pré-concentração em coluna cromatográfica de troca catiônica ou por co-precipitação com oxalato de cálcio e óxido de ferro 
contínuas, sendo assim foram consideradas peças fundamentais ao desenvolvimento da AAS

Assim como as lâmpadas de catodo oco, o sistema composto de fonte contínua, monocromadores de alta resolução e detectores de estado sólido marcaram a história da espectrometria de absorção atômica. Os avanços obtidos com este sistema, dentre eles o alto poder de resolução, a melhoria da razão sinal/ruído e o uso de apenas uma fonte de radiação cobrindo toda a faixa espectral de uso na AAS convencional e mais outras linhas do espectro necessárias à determinação de elementos cujas fontes de linhas não tinham ainda sido confeccionadas, abrem novas possibilidades para a AAS. Outra conquista de grande importância foi o aumento significativo da frequiência analítica obtida com o desenvolvimento de instrumentos capazes de realizar determinações multi-elementares seqüenciais em FAAS.

Aliado às tão conhecidas qualidades da AAS, a exemplo da simplicidade, do baixo custo de operação e da baixa possibilidade de interferências espectrais, todo o avanço recentemente alcançado reafirma a AAS como uma importante técnica de determinação elementar de metais e alguns ametais. Tais avanços incluem a aplicação de novos procedimentos para introdução de amostras em suspensão, sólidas e na fase gasosa $;{ }^{76} \mathrm{o}$ uso de sistemas alternativos de atomização, como o filamento de tungstênio e o forno-filtro com aquecimento transversal $;{ }^{77}$ fontes contínuas de radiação; sistemas de alta resolução, abrangendo os arranjos de detectores de carga acoplada ${ }^{78}$ e detectores de estado sólido $;{ }^{79}$ utilização de padronização interna para minimizar erros e possíveis efeitos interferentes. ${ }^{80-84}$

Mesmo havendo desafios a serem superados na determinação multi-elementar, a AAS alcançou avanços que mais uma vez a colocam nos rumos para suprir os anseios da química moderna.

\section{AGRADECIMENTOS}

Ao Conselho Nacional de Desenvolvimento Científico e Tecnológico $(\mathrm{CNPq})$, à Fundação de Amparo à Pesquisa do Estado da Bahia (FAPESB), à Coordenação de Aperfeiçoamento de Pessoal de Nível Superior (CAPES) e à Financiadora de Estudos e Projetos (FINEP) pelo apoio e suporte financeiro.

\section{REFERÊNCIAS}

1. Welz, B.; Sperling, M.; Atomic Absorption Spectrometry, $3^{\text {rd }}$ ed., WileyVCH: Weinheim, 1999.

2. Sneddon, J.; Smith, M. V.; Industhy, S.; Lee, Y.; Spectroscopy 1995, 10 , 26

3. Walsh, A.; Spectrochim. Acta, Part B 1955, 7, 108.

4. Alkemade, C. T. J.; Milatz, J. M. W.; J. Opt. Soc. Am. B 1955, 45, 583.

5. Koirtyohann, S. R.; Spectrochim. Acta, Part B 1980, 35, 663.

6. Walsh, A.; Anal. Chem. 1991, 63, 933A

7. L'vov, B.; Anal. Chem. 1991, 63, 924A.

8. Slavin, W.; Anal. Chem. 1991, 63, 1033A.

9. Walsh, A.; Spectrochim. Acta, Part B 1999, 54, 1943.

10. Walsh, A.; Spectrochim. Acta, Part B 1980, 35, 643.

11. Skoog, D. A.; Holler, F. J.; Nieman, T. A.; Princípios de Análise Instrumental, $5^{\mathrm{a}}$ ed., Bookman: Porto Alegre, 2002.

12. Sneddon, J.; Farah, B. D.; Farah, K. S.; Microchem. J. 1993, 48, 318.

13. Filgueras, C. A. L.; Química Nova na Escola 1996, n 3, 22.

14. Welz, B.; Becker-Ross, H.; Florek, S.; Heitmann, U.; Vale, M. G. R.; J. Braz. Chem. Soc. 2003, 14, 220.

15. L'vov, B.; Spectrochim. Acta, Part B 1961, 17, 108.

16. Welz, B.; Spectrochim. Acta, Part B 1999, 54, 2081.

17. Lowe, R. M.; Sullivan, J.V.; Spectrochim. Acta, Part B 1999, 54, 2031.

18. Slavin, W.; Maninng, D. C.; Carnrick, G. R.; At. Spectrosc. 1981, 2, 137.
19. Sneddon, J.; Farah, K. S.; Advances in Atomic Spectroscopy, 3, JAI Press Inc.: Louisiana, 1997.

20. Boar, P. L.; Spectrochim. Acta, Part B 1999, 54, 1989.

21. Hieftje, G. M.; J. Anal. At. Spectrom. 1989, 4, 117.

22. Aldous, K. M.; Mitchell, D. G.; Jackson, K. W.; Anal. Chem. 1975, 47, 7.

23. Harnly, J. M.; J. Anal. At. Spectrom. 1998, 14, 137.

24. Harnly, J. M.; O'Haver, T. C.; Golden, B.; Wolf, W. R.; Anal. Chem. 1979, 51, 2007.

25. Harnly, J. M.; Miller-Ihli, N.; O'Haver, T. C.; Spectrochim. Acta, Part B 1984, 39, 305

26. Harnly, J. M.; Anal. Chem. 1986, 58, 933A.

27. Harnly, J. M.; Fresenius J. Anal. Chem. 1996, 355, 501.

28. Carroll, J.; Miller-Ihli, N. J.; Harly, J. M.; Littlejohn, D.; Ottaway, J. M.; O'Haver, T. C.; Analyst 1985, 110, 1153.

29. Farah, K.; Sneddon, J.; Talanta 1993, 40, 879.

30. Sneddon, J.; Thiem, T. L.; Lee, Y.; Microchem. J. 1993, 48, 65.

31. Deval, A.; Sneddon, J.; Microchem. J. 1995, 52, 96.

32. Lee, Y. I.; Smith, M. V.; Indurthy, S.; Deval, A.; Sneddon, J.; Spectrochim. Acta, Part B 1996, 51, 109.

33. Lee, Y. I.; Indurthy, S.; Smith, M. V.; Sneddon, J.; Anal. Lett. 1996, 29 , 2515.

34. Hoenig, M.; Cilissen, A.; Spectrochim. Acta, Part B 1997, 52, 1443.

35. Hammond, J. L.; Lee, Y.; Noble, C. N.; Beck, J. N.; Proffitt, C. E.; Sneddon, J.; Talanta 1998, 47, 261.

36. Gomes Neto, J. A.; Freschi, G. P. G.; Dakuzaku, C. S.; Moraes, M.; Nóbrega, J. A.; Spectrochim. Acta, Part B 2001, 56, 1987.

37. Myöhänen, T.; Mäntylahti, V.; Koivunen, K.; Matilainen, R.; Spectrochim. Act, Part B 2002, 57, 1681.

38. Oliveira, P. V.; Correia, P. R. M.; Oliveira, E.; Talanta 2002, 57, 527.

39. Oliveira, P. V.; Oliveira, E.; Fresenius J. Anal. Chem. 2001, 371, 909.

40. Carrión, N.; Itriago, A. M.; Alvarez, M. A.; Eljuri, E.; Talanta 2003, 61, 621.

41. Chiu, C.; Sung, Y.; Huang, S.; Spectrochim. Acta, Part B 2003, 58, 575.

42. Nomura, C. S.; Correia, P. R. M.; Oliveira, P. V.; Oliveira, E.; J. Braz. Chem. Soc. 2004, 15, 75 .

43. Zheng, C. B.; He, Y. H.; Wei, S. Y.; Hou, X. D.; J. Anal. At. Spectrom. 2005, 20, 60 .

44. Pancras, J. P.; Ondov, J. M.; Zeisler, R.; Anal. Chim. Acta 2005, 538, 303.

45. Minami, H.; Yada, M.; Yoshida, T.; Zhang, Q. B.; Inoue, S.; Atsuya, I.; Anal. Sci. 2004, 20, 455.

46. Oliveira, A. P.; Gomes Neto, J. A. G.; Nóbrega, J. A.; Oliveira, P. V.; At. Spectrosc. 2005, 26, 158

47. Correia, P. R. M.; Oliveira, P. V.; Talanta 2005, 67, 46.

48. Oliveira, A. P.; Gomes Neto, J. A.; Nóbrega, J. A.; Oliveira, P. V.; Spectrochim. Acta, Part B 2005, 60, 681 .

49. Freschia, C. D.; Freschia, G. P. G.; Gomes Neto, J. A.; Nóbrega, J. A.; Oliveira, P. V.; Spectrochim. Acta, Part B 2005, 60, 759.

50. Fernandes, K. G.; de Moraes, M.; Gomes Neto, J. A.; Nóbrega, J. A.; Oliveira, P. V.; Analyst 2002, 127, 157.

51. Correia, P. R. M.; Nomura, C. S.; Oliveira, P. V.; Anal. Sci. 2003, 19, 1523.

52. Bencs, L.; Szakacs, O.; Kantor, T.; Varga, I.; Bozsai, G.; Spectrochim. Acta, Part B 2000, 55, 883 .

53. Oliveira, A. P.; de Moraes, M.; Gomes Neto, J. A.; Lima, E. C.; At. Spectrosc. 2002, 23, 190.

54. Radziuk, B.; Romanova, N. P.; Thomassen, Y.; Anal. Communications 1999, 36, 13.

55. Salido, A.; Jones, B. T.; Talanta 1999, 50, 649.

56. Su, P. G.; Huang, S. D.; J. Anal. At. Spectrom. 1998, 13, 641.

57. White, M. A.; Panayi, A.; At. Spectrosc. 1998, 19, 89. 
58. Iversen, B. S.; Panayi. A.; Camblor, J. P.; Sabbioni, E.; J. Anal. At. Spectrom. 1996, 11, 594.

59. Garbos, S.; Walcerz, M.; Bulska, E.; Hulanicki, A.; Spectrochim. Acta, Part B 1995, 50, 1669.

60. Sengupta, J. G.; Talanta 1993, 40, 791

61. Gupta, J. G. S.; J. Anal. At. Spectrom. 1993, 8, 93.

62. Freschi, G. P. G.; Dakuzaku, C. S.; Gomes Neto, J. A.; Moraes, M.; Eclética Química 2000, 25, 213.

63. Welz, B.; Becker-Ross, H.; Florek, S.; Heitmenn, U.; High-Resolution Continuum Source AAS-The Better Way to Do Atomic Absorptio Spectrometry, Wiley-VCH: Weinheim, 2005.

64. Welz, B.; Anal. Bioanal. Chem. 2005, 381, 69.

65. Projahn, H. D.; Steeg U.; Sanders, J.; Vanciay E.; Anal. Bioanal. Chem. 2004, 378, 1083.

66. Wang, A. X.; Zhang,Z. Y.; Lu, J. J.; Cui, X. Y.; Spectroscopy and Spectral Analysis 2003, 23, 785.

67. Wang, A. X.; Guo, L. P.; Zhang, H.; Chin. J. Anal. Chem. 2005, 33, 385 .

68. Niedzielski, P.; Anal. Chim. Acta 2005, 551, 199.

69. Lobo, I. P.; Dissertação de Mestrado, Universidade Federal da Bahia, Brasil, 2005.

70. Amorim, F. A. C.; Dissertação de Mestrado, Universidade Federal da Bahia, Brasil, 2004.

71. Amorim, F. A. C.; Ferreira, S. L. C.; Talanta 2005, 65, 960.
72. da Silva, E. G. P.; Santos, A. C. D.; Costa, A. C. S.; Fortunato, D. M. D.; Jose, N. M.; Korn, M. G. A.; dos Santos, W. N. L; Ferreira, S. L. C.; Microchem. J. 2006, 82, 159.

73. Amorim, F. A. C.; Lima, D. C.; dos Santos, D. S. S.; Teixeira, L. S. G.; Ferreira, S. L. C.; Resumos da $29^{a}$ Reunião Anual da Sociedade Brasileira de Química, Águas de Lindóia, Brasil, 2006.

74. da Silva, J. E.; da Silva, F. A.; Pimentel, M. F.; Honorato, R. S.; da Silva, V. L.; Montenegro, B. S. M.; Araujo, A. N.; Talanta 2006, 70, 522.

75. Ferreira, S. L. C.; Souza, A. S.; Brandão, G. C.; Ferreira, H. S.; dos Santos, W. N. L.; Pimentel, M. F.; Vale, M. G. R.; Talanta 2007, no prelo, doi: 10.1016/j.talamta.2007.06.038.

76. Nakahara, T.; Anal. Sci. 2005, 21, 477.

77. Heitmann, U.; Becker-Ross, H.; Katskov, D.; Spectrochim Acta, Part B 2006, 61, 351 .

78. Harnly, J. M.; J. Anal. At. Spectrom. 1999, 14, 137.

79. Radziuk, B.; Rodel, G.; Zeiher, M.; Mizuno, S.; Yamamoto, K.; J. Anal. At. Spectrom. 1995, 10, 415.

80. Takada, T.; Nakano, K.; Anal. Chim. Acta 1979, 107, 129.

81. Takada, T.; Nakano, K.; Spectrochim. Acta, Part B 1981, 36, 735.

82. Fernandes, K. G.; de Moraes, M.; Gomes Neto, J. A.; Nóbrega, J. A.; Oliveira, P. V.; Quim. Nova 2003, 26, 249.

83. Oliveira, A. P.; Gomes Neto, J. A.; Nóbrega, J. A.; Oliveira, P. V.; Talanta 2004, 64, 334.

84. Correia, P. R. M.; Oliveira, P. V.; Quim. Nova 2005, 28, 539. 